\title{
Analysis of $W^{ \pm}+4 \gamma$ in the 2HDM Type-I at the LHC
}

\section{Yan Wang, ${ }^{a}$ A. Arhrib, ${ }^{b}$ R. Benbrik, ${ }^{c}$ M. Krab, ${ }^{d}$ B. Manaut, ${ }^{d}$ S. Moretti ${ }^{e}$ and Qi-Shu Yan ${ }^{f, g}$}

${ }^{a}$ College of Physics and Electronic Information, Inner Mongolia Normal University, Hohhot 010022, P.R. China

${ }^{b}$ Abdelmalek Essaadi University, Faculty of Sciences and Techniques,

B.P. 2117 Tétouan, Tanger, Morocco

${ }^{c}$ Laboratoire de Physique Fondamentale et Appliquée de Safi, Faculté Polydisciplinaire de Safi, Sidi Bouzid, B.P. 4162, Safi, Morocco

${ }^{d}$ Polydisciplinary Faculty, Laboratory of Research in Physics and Engineering Sciences, Team of Modern and Applied Physics, Sultan Moulay Slimane University, Beni Mellal 23000, Morocco

${ }^{e}$ School of Physics and Astronomy, University of Southampton, Southampton SO17 1BJ, U.K.

${ }^{f}$ Center for Future High Energy Physics, Chinese Academy of Sciences, Beijing 100049, P.R. China

${ }^{g}$ School of Physics Sciences, University of Chinese Academy of Sciences, Beijing 100039, P.R. China

E-mail: wangyan@imnu.edu.cn, aarhrib@gmail.com, r.benbrik@uca.ma, mohamed.krab@usms.ac.ma, b.manaut@usms.ma, s.moretti@soton.ac.uk, yanqishu@ucas.ac.cn 
AbStract: We analyse a light charged Higgs boson in the 2-Higgs Doublet Model (2HDM) Type-I, when its mass satisfies the condition $M_{H^{ \pm}}<M_{t}+M_{b}$ and the parameter space is consistent with theoretical requirements of self-consistency as well as the latest experimental constraints from Large Hadron Collider (LHC) and other data. Over such a parameter space, wherein the Standard Model (SM)-like state discovered at the LHC in 2012 is the heaviest CP-even state of the $2 \mathrm{HDM}$, it is found that the decay modes of the charged Higgs boson are dominated by $H^{ \pm} \rightarrow W^{ \pm(*)} h$. Furthermore, the light neutral Higgs boson $h$ dominantly decays into two photons. Under these conditions, we find that the production and decay process $p p \rightarrow H^{ \pm} h \rightarrow W^{ \pm(*)} h h \rightarrow \ell \nu_{\ell}+4 \gamma(\ell=e, \mu)$ is essentially background free. However, since the $W^{ \pm(*)}$ could be largely off-shell and the $h$ state is very light, so that both the lepton coming from the former and the photons coming from the latter could be rather soft, we perform here a full Monte Carlo (MC) analysis at the detector level demonstrating that such a $W^{ \pm}+4 \gamma$ signal is very promising, as it would be yielding significant excesses at the LHC with an integrated luminosity of $L=300 \mathrm{fb}^{-1}$ at both $\sqrt{s}=13$ and $14 \mathrm{TeV}$.

Keywords: Beyond Standard Model, Higgs Physics

ArXiv EPrint: 2107.01451 


\section{Contents}

1 Introduction 1

2 The 2 HDM 2

2.1 Constraints on the 2HDM 3

2.2 Parameter space scans 5

3 Collider phenomenology 6

3.1 Event generation 6

$\begin{array}{lll}3.2 & \text { Event reconstruction } & 7\end{array}$

3.3 Significances 8

4 Conclusions 13

\section{Introduction}

The discovery of a $125 \mathrm{GeV}$ scalar particle at the LHC represents the last piece of the Standard Model (SM). Generally speaking, an agreement between the measured and predicted properties of this particle has been reached at a $2 \sigma$ level. But it is still interesting to examine the possibility of whether the Higgs sector can include more (pseudo)scalar particles, which is quite natural in those new physics models with extra doublets or triplets. One of the typical feature of these new physics models is that they predict one or more charged Higgs bosons. Thus, if a charged Higgs boson could be found at the LHC, it would be a clear evidence of new physics Beyond the SM (BSM).

One of the simplest extensions of the SM is the 2HDM, which contains two complex Higgs doublets. After Electro-Weak Symmetry Breaking (EWSB), there are 3 Goldstone bosons, which are 'eaten' by the $W^{ \pm}$and $Z$ bosons, and 5 degrees of freedom which incarnate 5 physical Higgs bosons. The latter are known as 2 neutral CP-even scalars $(h$ and $H$, with $M_{h}<M_{H}$ ), a CP-odd pseudoscalar $(A)$ and two charged Higgs states $H^{ \pm}$.

In order to satisfy the stringent experimental constraints from Flavour Changing Neutral Currents (FCNCs) at the tree level, typically, a $Z_{2}$ symmetry is introduced into the Yukawa sector such that each type of fermion only couples to one of the doublets of the 2HDM. Depending on the $Z_{2}$ charge assignment of the Higgs doublets, we can define four basic scenarios, known as (Yukawa) Types. In the Type-I scenario, all fermions couple to the second Higgs doublet (labelled as $\Phi_{2}$ ) while the tree-level Yukawa couplings to the first Higgs doublet $\Phi_{1}$ are vanishing. In such a scenario, a light charged Higgs boson (say, lighter than $M_{t}-M_{b}$ ) is still allowed by even the most stringent bounds (from direct $H^{ \pm}$ searches and $B$ physics measurements). 
In a recent study [1], it was found that in the Type-I scenario of the $2 \mathrm{HDM}$, the decay mode $H^{ \pm} \rightarrow W^{ \pm(*)} h$ could become the dominant one, in the so-called fermiophobic limit of the 2HDM Type-I. In these conditions, the main production and decay process of a $H^{ \pm}$state and, consequently, the emerging experimental signatures could be different from what the ATLAS and CMS Collaborations are looking for, which is the process $p p \rightarrow t \bar{t} \rightarrow$ $W^{\mp} H^{ \pm} b \bar{b}$ with $H^{ \pm}$dominantly decaying into a pair of fermions $(\tau \nu$ and $c s)$. At the same time, when also the $h$ state is fermiophobic, its decay modes into SM fermions could be highly suppressed, which results into a large Branching Ratio (BR) for the mode $h \rightarrow \gamma \gamma$. therefore, in [1], it was found that the associated production process $p p \rightarrow H^{ \pm} h$ could lead to a potentially detectable $W^{ \pm}+4 \gamma$ final state. According to the parton level analysis in [1], it was observed that this signature is almost background free and could have a large significance. Therefore, it is worth to examine whether this statement is robust enough after taking into account parton shower, hadronisation, heavy flavour decays and detector effects.

In this paper, like in [1], we assume that the heaviest CP-even Higgs boson $H$ is the observed SM-like Higgs boson, which properties are consistent with measurements at the LHC. Furthermore, due to the constraints from EW precision tests, it is noted that the lighter Higgs boson $h$ can be lighter than $125 \mathrm{GeV}$. In such a parameter space, a light charged Higgs boson $H^{ \pm}$is thus accompanied by a light Higgs boson $h$. We focus our collider phenomenology study on the signal process $p p \rightarrow H^{ \pm} h \rightarrow W^{ \pm(*)} h h \rightarrow \ell \nu_{\ell}+4 \gamma(\ell=e, \mu)$ and examine its feasibility at the LHC. It will eventually be found that, after taking into account theoretical and experimental constraints, there are points in the 2HDM Type-I parameter space which can be either discovered or ruled out already with the current integrated luminosity at the LHC and that, with the full Run 3 data set, or a tenth of the High-Luminosity LHC (HL-LHC) one [2, 3], a definite statement on this BSM scenario can be made.

The paper is organised as follows. In section 2, we briefly describe the $2 \mathrm{HDM}$ and its Yukawa scenarios, then introduce a few Benchmark Points (BPs) for our MC analysis which pass all present constraints, both theoretical and experimental. In section 3, we perform a detailed collider analysis of these BPs and examine the potential to discover the aforementioned signature of this $2 \mathrm{HDM}$ Type-I scenario. In section 4, we present some conclusions.

\section{The $2 \mathrm{HDM}$}

The scalar sector of the $2 \mathrm{HDM}$ contains two complex SU(2) doublets with hypercharge $Y=+1$,

$$
\Phi_{a}=\left(\begin{array}{c}
\phi_{a}^{+} \\
\phi_{a}^{0}
\end{array}\right), \quad\left\langle 0\left|\Phi_{a}\right| 0\right\rangle=\left(\begin{array}{c}
0 \\
v_{a} / \sqrt{2}
\end{array}\right) \quad(a=1,2)
$$

where $v_{1}$ and $v_{2}$ are the Vacuum Expectation Values (VEVs) of the neutral Higgs field components that break spontaneously the EW gauge symmetry to the Electro-Magnetic $(\mathrm{EM})$ one, $\mathrm{SU}(2)_{L} \otimes \mathrm{U}(1)_{Y} \rightarrow \mathrm{U}(1)_{\mathrm{EM}}$. The most general $\mathrm{SU}(2)_{L} \times \mathrm{U}(1)_{Y}$ invariant scalar 


\begin{tabular}{|c|c|c|c|}
\hline$\phi$ & $\xi_{\phi}^{u}$ & $\xi_{\phi}^{d}$ & $\xi_{\phi}^{\ell}$ \\
\hline$h$ & $\cos \alpha / \sin \beta$ & $\cos \alpha / \sin \beta$ & $\cos \alpha / \sin \beta$ \\
$H$ & $\sin \alpha / \sin \beta$ & $\sin \alpha / \sin \beta$ & $\sin \alpha / \sin \beta$ \\
$A$ & $\cot \beta$ & $-\cot \beta$ & $-\cot \beta$ \\
\hline
\end{tabular}

Table 1. Yukawa couplings of the $h, H$ and $A$ bosons to quarks and leptons in the Type-I 2HDM.

potential involving two Higgs doublets can be written as [4]:

$$
\begin{aligned}
V\left(\Phi_{1}, \Phi_{2}\right)= & m_{11}^{2} \Phi_{1}^{\dagger} \Phi_{1}+m_{22}^{2} \Phi_{2}^{\dagger} \Phi_{2}-\left[m_{12}^{2} \Phi_{1}^{\dagger} \Phi_{2}+\text { h.c. }\right] \\
& +\frac{\lambda_{1}}{2}\left(\Phi_{1}^{\dagger} \Phi_{1}\right)^{2}+\frac{\lambda_{2}}{2}\left(\Phi_{2}^{\dagger} \Phi_{2}\right)^{2}+\lambda_{3}\left(\Phi_{1}^{\dagger} \Phi_{1}\right)\left(\Phi_{2}^{\dagger} \Phi_{2}\right)+\lambda_{4}\left(\Phi_{1}^{\dagger} \Phi_{2}\right)\left(\Phi_{2}^{\dagger} \Phi_{1}\right) \\
& +\left\{\frac{\lambda_{5}}{2}\left(\Phi_{1}^{\dagger} \Phi_{2}\right)^{2}+\left[\lambda_{6}\left(\Phi_{1}^{\dagger} \Phi_{1}\right)+\lambda_{7}\left(\Phi_{2}^{\dagger} \Phi_{2}\right)\right] \Phi_{1}^{\dagger} \Phi_{2}+\text { h.c. }\right\}
\end{aligned}
$$

The hermiticity of the potential requires all parameters to be real except $m_{12}^{2}, \lambda_{5}, \lambda_{6}$ and $\lambda_{7}$. For simplicity, we will work with a CP-conserving scalar potential by choosing $m_{12}^{2}$ and $\lambda_{5,6,7}$ to be real. Note that, in $Z_{2}$ symmetric models, terms that are proportional to $\lambda_{6}$ and $\lambda_{7}$ in the scalar potential are absent, to ensure the suppression of FCNCs at tree level (as already remarked upon).

The Yukawa Lagrangian, which describes the interactions between the (pseudo)scalar fields and the fermion sector, is given as follows [4]:

$$
\mathcal{L}_{Y}=\bar{Q}_{L}^{\prime}\left(Y_{1}^{u} \tilde{\Phi}_{1}+Y_{2}^{u} \tilde{\Phi}_{2}\right) u_{R}^{\prime}+\bar{Q}_{L}^{\prime}\left(Y_{1}^{d} \Phi_{1}+Y_{2}^{d} \Phi_{2}\right) d_{R}^{\prime}+\bar{L}_{L}^{\prime}\left(Y_{1}^{l} \Phi_{1}+Y_{2}^{l} \Phi_{2}\right) l_{R}^{\prime}+\text { h.c. }
$$

where $Q_{L}^{\prime}$ and $L_{L}^{\prime}$ are the weak isospin quark and lepton doublets, $u_{R}^{\prime}$ and $d_{R}^{\prime}$ denote the right-handed quark singlets and $Y_{1,2}^{u}, Y_{1,2}^{d}$ and $Y_{1,2}^{l}$ are coupling matrices in flavour space.

The implementation of the aforementioned discrete symmetry, depending on the $Z_{2}$ assignments, leads to four Types of 2HDM: commonly denoted as Type-I, -II, -X and -Y. In the mass eigenstate basis, their treatment can be unified in the following form [4]:

$$
\begin{aligned}
-\mathcal{L}_{Y}^{I}= & +\sum_{f=u, d, \ell}\left[m_{f} \bar{f} f+\left(\frac{m_{f}}{v} \xi_{h}^{f} \bar{f} f h+\frac{m_{f}}{v} \xi_{H}^{f} \bar{f} f H-i \frac{m_{f}}{v} \xi_{A}^{f} \bar{f} \gamma_{5} f A\right)\right] \\
& +\frac{\sqrt{2}}{v} \bar{u}\left(m_{u} V \xi_{A}^{u} P_{L}+V m_{d} \xi_{A}^{d} P_{R}\right) d H^{+}+\frac{\sqrt{2} m_{\ell} \xi_{A}^{\ell}}{v} \bar{\nu}_{L} \ell_{R} h^{+}+\text {h.c. },
\end{aligned}
$$

where $P_{L, R}=\left(1 \mp \gamma_{5}\right) / 2$ and $V$ denotes the Cabibbo-Kobayashi-Maskawa (CKM) matrix.

In our study, like in [1], we choose to focus on Type-I, where only one doublet $\Phi_{2}$ couples to all fermions and thus the Higgs-fermion couplings are flavour diagonal in the fermion mass basis and depend only on two angles, $\alpha$ (parameterising the mixing between $h$ and $H$ ) and $\beta$ (which tangent is given by the ratio of the two VEVs), as shown in table 1 .

\subsection{Constraints on the 2HDM}

There are certain theoretical restrictions and experimental constraints on the scalar potential that have to be imposed in order to obtain a viable realisation of the $2 \mathrm{HDM}$. We 
note that both theoretical consistency and experimental data have already limited the parameter space of the $2 \mathrm{HDM}$.

In our study, we consider the following theoretical constraints.

(1) Perturbativity is not invalidated in the Higgs sector, so long that $\lambda_{i}<4 \pi$ ( $i=$ $1, \ldots, 5)$.

(2) The $S$-matrix satisfies all relevant tree-level unitarity constraints, which implies that the quartic couplings $\lambda_{i}$ satisfy the following relations: [5]

$$
\begin{aligned}
3\left(\lambda_{1}+\lambda_{2}\right) \pm \sqrt{9\left(\lambda_{1}-\lambda_{2}\right)^{2}+4\left(2 \lambda_{3}+\lambda_{4} \mid\right)^{2}} & <16 \pi \\
\lambda_{1}+\lambda_{2} \pm \sqrt{\left(\lambda_{1}-\lambda_{2}\right)^{2}+4\left|\lambda_{5}\right|^{2}} & <16 \pi \\
\lambda_{1}+\lambda_{2} \pm \sqrt{\left(\lambda_{1}-\lambda_{2}\right)^{2}+4\left|\lambda_{5}\right|^{2}} & <16 \pi \\
\lambda_{3}+2 \lambda_{4} \pm 3\left|\lambda_{5}\right| & <8 \pi \\
\lambda_{3} \pm \lambda_{4} & <8 \pi, \\
\lambda_{3} \pm\left|\lambda_{5}\right| & <8 \pi .
\end{aligned}
$$

(3) The scalar potential is finite at large field values and contains no flat directions, which translate into the bounds [6]:

$$
\lambda_{1,2}>0, \lambda_{3}>-\sqrt{\lambda_{1} \lambda_{2}}, \lambda_{3}+\lambda_{4}-\left|\lambda_{5}\right|>-\sqrt{\lambda_{1} \lambda_{2}} .
$$

On the experimental side, we consider the following constraints.

(4) Limits from the EW oblique parameters $S, T$ and $U$ [7], for which we check their consistency at $95 \%$ Confidence Level (CL) with the following measurements [8]:

$$
S=0.05 \pm 0.11, \quad T=0.09 \pm 0.13, \quad U=0.01 \pm 0.11
$$

(5) To study the effects of LEP, TeVatron and LHC data affecting the Higgs sector, we have considered both exclusions from nil searches for Higgs boson companions, via HiggsBounds-5.9.0 [9], and measurements of the SM-like Higgs boson properties, via HiggsSignals-2.6.0 [10] (for which we have enforced a best fit at 95.5\% CL, which corresponds to $\left.\Delta \chi^{2}\left(\chi^{2}-\chi_{\min }^{2}\right) \leq 5.99\right)$.

(6) Constraints from $B$ physics observables, which give rise to bounds on the parameter space of the $2 \mathrm{HDM}$, as per the measured values in table 2 (where we also give the corresponding SM predictions).

(Note that we have used SuperIso v4.1 [13] to compute the exclusions from flavour physics observables and 2HDMC [14] to check the theoretical constraints as well as the parameters $S, T$ and $U$.)

The results of related theoretical analyses and experimental measurements from LEP and Tevatron were also taken into consideration here [15-24]. But these studies are mainly 


\begin{tabular}{|c|c|c|}
\hline Observable & Experimental result & SM prediction \\
\hline $\mathrm{BR}\left(B \rightarrow X_{s} \gamma\right)$ & $(3.32 \pm 0.15) \times 10^{-4}[11]$ & $(3.34 \pm 0.22) \times 10^{-4}$ \\
\hline $\mathrm{BR}\left(B_{s} \rightarrow \mu^{+} \mu^{-}\right)$ & $(3.0 \pm 0.6 \pm 0.25) \times 10^{-9}[12]$ & $(3.54 \pm 0.27) \times 10^{-9}$ \\
\hline $\mathrm{BR}\left(B_{d} \rightarrow \tau \nu\right)$ & $(1.06 \pm 0.19) \times 10^{-4}[11]$ & $(0.82 \pm 0.29) \times 10^{-4}$ \\
\hline
\end{tabular}

Table 2. Experimental results and SM predictions for selected flavour observables.

focused on exactly fermiophobic models, while in our work we are interested in the parameter spaces only close to the fermiophobic limit, so that the stringent constraints stemming from those papers cannot generally be extended to our case. For example, in ref. [21], the authors studied the same $W^{ \pm}+4 \gamma$ process as here but in the exact fermiophobic limit and suggested several plans to search for it at the Tevatron. In ref. [22], the CDF collaboration reported a search for at least three photons final states for $M_{H}^{ \pm}$values ranging from 30 to $300 \mathrm{GeV}$, based on the idea from ref. [21]. There was no evidence of such signal. However, since only $9.2 \mathrm{fb}^{-1}$ data were used at $1.96 \mathrm{TeV}$ collider, such results do not constrain our following BPs. (A detailied discussion of this can be found in [1].)

\subsection{Parameter space scans}

In this work, we concentrate on the scenario in which the $h$ state is fermiophobic, which occurs near the alignment limit $\sin (\beta-\alpha) \sim 0$. In such a limit, all fermionic decays of the lightest CP-even Higgs boson are suppressed, so that $h \rightarrow \gamma \gamma$ can become significant. In the SM, the $\gamma \gamma$ decay of the Higgs boson is generated by the dominant $W^{ \pm}$loop and the subdominant top quark one, which have opposite signs and thus cancel one another somewhat. In the $2 \mathrm{HDM}$, the additional $H^{ \pm}$loop also contributes. In the Type-I case, one has the following coupling dependencies for the lightest CP-even state: $h W^{+} W^{-} \sim$ $\sin (\beta-\alpha), h q \bar{q} \sim \cos \alpha / \sin \beta$ while the $h H^{+} H^{-}$vertex is given by the parameters of the scalar potential. Since, for fermionic loops, the coupling is proportional to $\cos \alpha$ and

$$
\cos \alpha=\sin \beta \sin (\beta-\alpha)+\cos \beta \cos (\beta-\alpha),
$$

when $\sin (\beta-\alpha)$ is negative and $\cos (\beta-\alpha)$ is positive, $\cos \alpha$ will be cancelled for a particular $\tan \beta$, which is when $h$ becomes fermiophobic and $h \rightarrow \gamma \gamma$ is enhanced because the aforementioned cancellation no longer occurs.

A numerical scan of the 2HDM Type-I parameters was performed in ref. [1], which satisfied all theoretical and experimental constraints mentioned above. Based on the same scan, in this work, we propose the following 14 BPs given in table 3. There are three comments to make on these 14 BPs.

- The mass of the charged Higgs boson can vary from $91.49 \mathrm{GeV}$ to $168.69 \mathrm{GeV}$. The CP-even Higgs boson $h$ is always lighter than $125 \mathrm{GeV}$ and lighter than the $H^{ \pm}$state. The $W^{ \pm}$boson from the charged Higgs boson decaying via $H^{ \pm} \rightarrow W^{ \pm(*)} h$ could be either on-shell or off-shell. If it is off-shell, the charged lepton emerging from it might be soft (as already remarked upon), like in BP4-BP10. 


\begin{tabular}{|c|c|c|c|c|c|c|c|c|}
\hline & $M_{h}$ & $M_{A}$ & $M_{H^{ \pm}}$ & $\sin (\beta-\alpha)$ & $\tan \beta$ & $m_{12}^{2}$ & $\sigma_{13}(W+4 \gamma)(\mathrm{fb})$ & $\sigma_{14}(W+4 \gamma)(\mathrm{fb})$ \\
\hline BP1 & 25.57 & 72.39 & 111.08 & -0.074 & 13.58 & 11.97 & 101.40 & 112.55 \\
\hline BP2 & 35.12 & 111.24 & 151.44 & -0.076 & 13.32 & 16.66 & 167.75 & 186.20 \\
\hline BP3 & 45.34 & 162.07 & 128.00 & -0.136 & 7.57 & 80.96 & 10.76 & 11.93 \\
\hline BP4 & 53.59 & 126.09 & 91.49 & -0.127 & 8.00 & 51.16 & 27.05 & 29.88 \\
\hline BP5 & 63.13 & 85.59 & 104.99 & -0.055 & 18.10 & 190.24 & 179.31 & 198.61 \\
\hline BP6 & 65.43 & 111.43 & 142.15 & -0.087 & 11.52 & 325.36 & 174.49 & 194.30 \\
\hline BP7 & 67.82 & 79.83 & 114.09 & -0.111 & 8.94 & 326.32 & 177.72 & 197.23 \\
\hline BP8 & 69.64 & 195.73 & 97.43 & -0.111 & 8.86 & 357.10 & 196.04 & 217.18 \\
\hline BP9 & 73.18 & 108.69 & 97.34 & -0.122 & 8.06 & 594.64 & 193.56 & 214.57 \\
\hline BP10 & 84.18 & 115.26 & 148.09 & -0.067 & 14.82 & 473.88 & 61.92 & 68.98 \\
\hline BP11 & 68.96 & 200.84 & 155.40 & -0.112 & 8.64 & 531.46 & 62.02 & 69.14 \\
\hline BP12 & 71.99 & 91.30 & 160.10 & -0.104 & 9.74 & 472.22 & 58.99 & 65.80 \\
\hline BP13 & 74.08 & 102.49 & 163.95 & -0.092 & 10.56 & 503.74 & 55.58 & 62.04 \\
\hline BP14 & 81.53 & 225.76 & 168.69 & -0.101 & 9.75 & 501.29 & 51.85 & 57.91 \\
\hline
\end{tabular}

Table 3. Input parameters and the Leading Order (LO) cross sections at the parton-level with $\sqrt{s}=13(14) \mathrm{TeV}$ for each BP are presented. All masses are in $\mathrm{GeV}$ and recall that $M_{H}=125 \mathrm{GeV}$.

- The parameter $\sin (\beta-\alpha)$ is constrained by the SM-like Higgs boson data from the LHC via the measurements of $H \rightarrow W^{ \pm} W^{\mp(*)}$ and $Z Z^{*}$, since the BRs of these modes are proportional to $\cos ^{2}(\beta-\alpha)$. Current LHC Higgs data essentially demand that $\left|\sin ^{2}(\beta-\alpha)=1-\cos ^{2}(\beta-\alpha)\right|<0.10$, which leads to a range of $|\sin (\beta-\alpha)|<0.3$ or so.

- For these BPs, the main production process of a charged Higgs boson is $p p \rightarrow H^{ \pm} h$, which cross section can be up to one order of magnitude larger than those of $p p \rightarrow$ $H^{ \pm} A$ and $p p \rightarrow H^{ \pm} H^{\mp}$, which are alternative discovery modes in this region of $2 \mathrm{HDM}$ Type-I parameter space [25-27]. Therefore, in the present analysis, we will focus on the $W^{ \pm}+4 \gamma$ signature stemming from the $p p \rightarrow H^{ \pm} h$ production process only.

Thus, since our signal is given by $p p \rightarrow H^{ \pm} h \rightarrow W^{ \pm(*)} h h \rightarrow \ell \nu_{\ell}+4 \gamma$, the dominant background processes are $W^{ \pm}+4 j 0 \gamma, W^{ \pm}+3 j 1 \gamma, W^{ \pm}+2 j 2 \gamma, W^{ \pm}+1 j 3 \gamma$ and $W^{ \pm}+0 j 4 \gamma$, where a jet has a certain probability to fake a photon.

\section{Collider phenomenology}

In this section, we present a detailed MC analysis at a detector level, including both signal and background events.

\subsection{Event generation}

Here we briefly describe MC event generation.

- We use MadGraph5_aMC@NLO v2.8.2 [28] (MG) to compute the cross sections and generate both signal and background events at parton level. We have adopted the 
following kinematic cuts (in pseudorapidity, transverse momentum and Missing $E_{T}$ (MET), where $E_{T}$ is the transverse energy (or momentum)) in order to improve the efficiency of the MC event generation

$$
|\eta(l, j, \gamma)|<2.5, \quad p_{T}(j, \gamma, l)>10 \mathrm{GeV}, \quad \Delta R(l, j, \gamma)>0.5, \quad \operatorname{MET}>5 \mathrm{GeV},
$$

where $j$ refers here to parton. The signal events are generated at LO, the cross sections for each BPs at the LHC with $\sqrt{s}=13(14) \mathrm{TeV}$ are listed in the last two column of table 3. The backgrounds are treated at LO, but this apparent inconsistency will become irrelevant once selection cuts are implemented, as the signal will be proven to be essentially backgound free for all BPs.

- After generating both signal and background events at the parton level, we pass them to Pythia v8 [29] to simulate initial and final state radiation (i.e., the QED and QCD emission), parton shower, hadronisation and heavy flavour decays.

- We use Delphes v3.4.2 [30] to simulate the detector effects. For each event, we cluster final particles into jets and we adopt the anti- $k_{t}$ jet algorithm [31] with jet parameter $\Delta R=0.5$ in the FastJet package [32]. ${ }^{1}$ Following the ATLAS analysis of [36], we will take as fake photon rate the value 0.001 , which describes the probability to mistag a jet as a photon.

Notice that, in the following, we will present event rates corresponding to an LHC energy of $\sqrt{s}=13 \mathrm{TeV}$ and $14 \mathrm{TeV}$ and integrated luminosity of $L=300 \mathrm{fb}^{-1}$.

\subsection{Event reconstruction}

The mass $M_{h}$ can be reconstructed on an event-by-event basis by pairing the four photons into two pairs by minimising the following $\chi^{2}$ :

$$
\chi^{2}=\left(M_{\gamma \gamma}^{1}-M_{h}\right)^{2}+\left(M_{\gamma \gamma}^{2}-M_{h}\right)^{2} .
$$

Obviously, there are 3 combinatorics for each event. When the combination which minimises the $\chi^{2}$ is found, we label the larger invariant mass of the pair of two photons as $M_{\gamma \gamma}^{1}$ and the other one is then labelled as $M_{\gamma \gamma}^{2}$. The distributions of these two reconstructed masses of $h$ for, e.g., BP5 are displayed in figure 1 , where $M_{\gamma \gamma}^{1}$ and $M_{\gamma \gamma}^{2}$ are close to $M_{h}$ at the same time.

The distribution of the reconstructed mass of the charged Higgs boson is shown in figure 2. Since there is missing energy, we use the standard method as the $W^{ \pm}$boson reconstruction. There are then two possible candidates for the light Higgs state, one is produced in the charged Higgs boson decay and the other is produced in association with it. Thus, we obtained two possible $H^{ \pm}$masses. As shown in figure 2 , the correct one is rather sharp, while the wrong one is more dispersed.

As demonstrated by figures 1 and 2, even though there is some amount of combinatorics, the salient kinematic features of the signal stem clearly in a variety of mass

\footnotetext{
${ }^{1}$ Results obtained from the $k_{t}$ [33] or Cambridge-Aachen [34, 35] algorithms are very similar.
} 


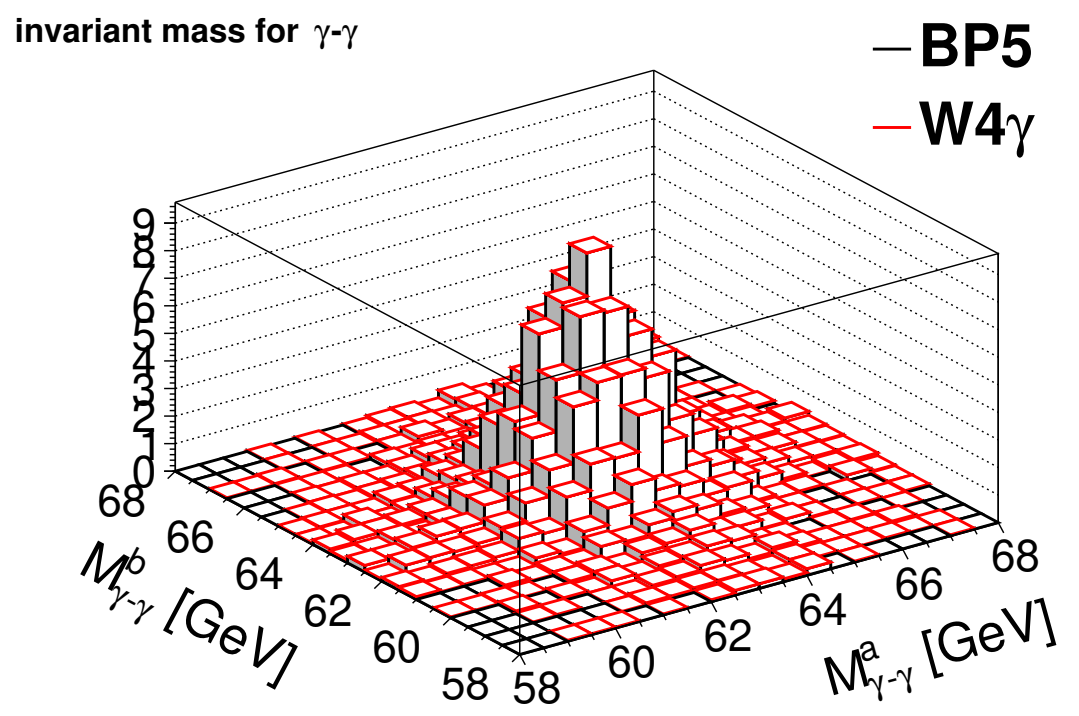

Figure 1. The 3D $M_{\gamma \gamma}$ distribution for the two $\gamma \gamma$ pairings of the BP5 signal.

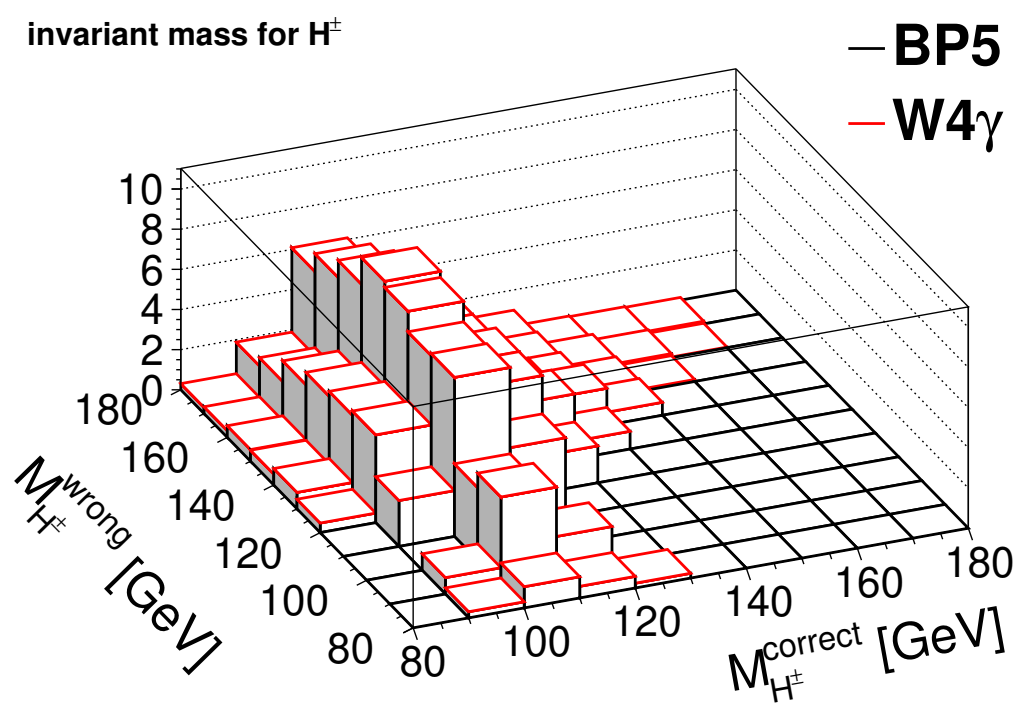

Figure 2. The 3D $M_{H^{ \pm}}$distribution for the two different combinations of the BP5 signal.

observables reconstructed from the $\ell \nu_{\ell}+4 \gamma$ final state, which can then help to distinguish between signal and background events or else characterise the former, depending on the size of the latter. For completeness, in figure 3, we show the MET distribution and total transverse momentum of all visible particles. The peaks of these two plots are at about $20 \mathrm{GeV}$, which means that one could add a further MET cut to suppress possible backgrounds from the $B$ hadron decays, though this should not be necessary, as we shall demonstrate next.

\subsection{Significances}

We now estimate the tagging efficiency for leptons and photons at detector level by using Delphes. We generate 10k events for each BP and count the percentage of events where 

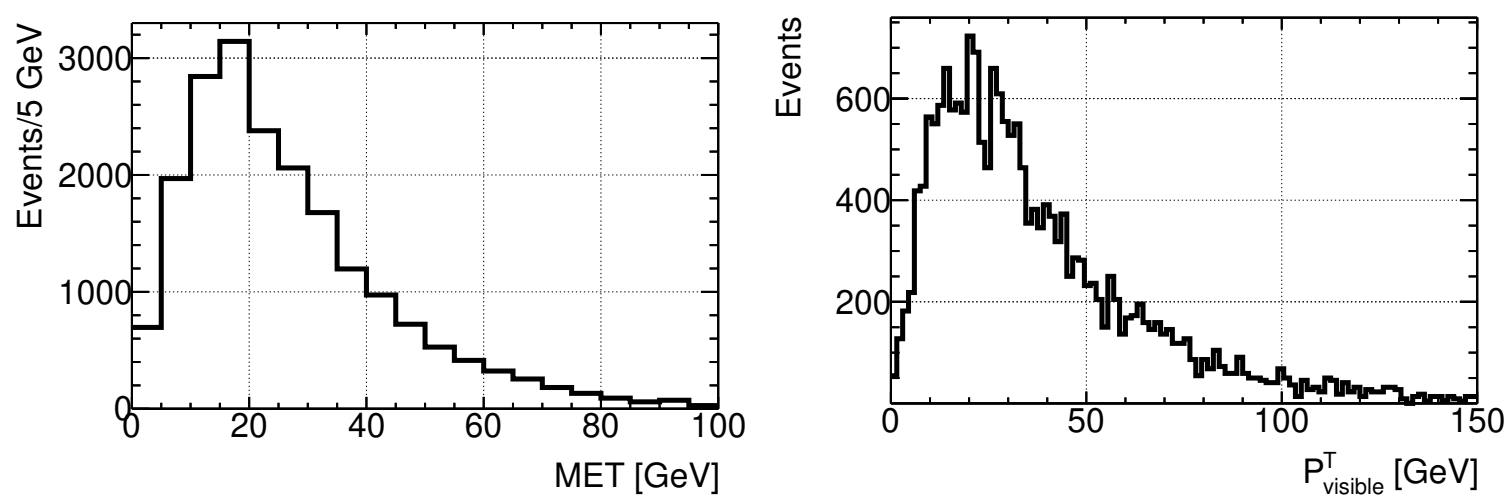

Figure 3. The MET (left) and the total transverse momentum of all visible particles (right) distributions for the BP4 signal.
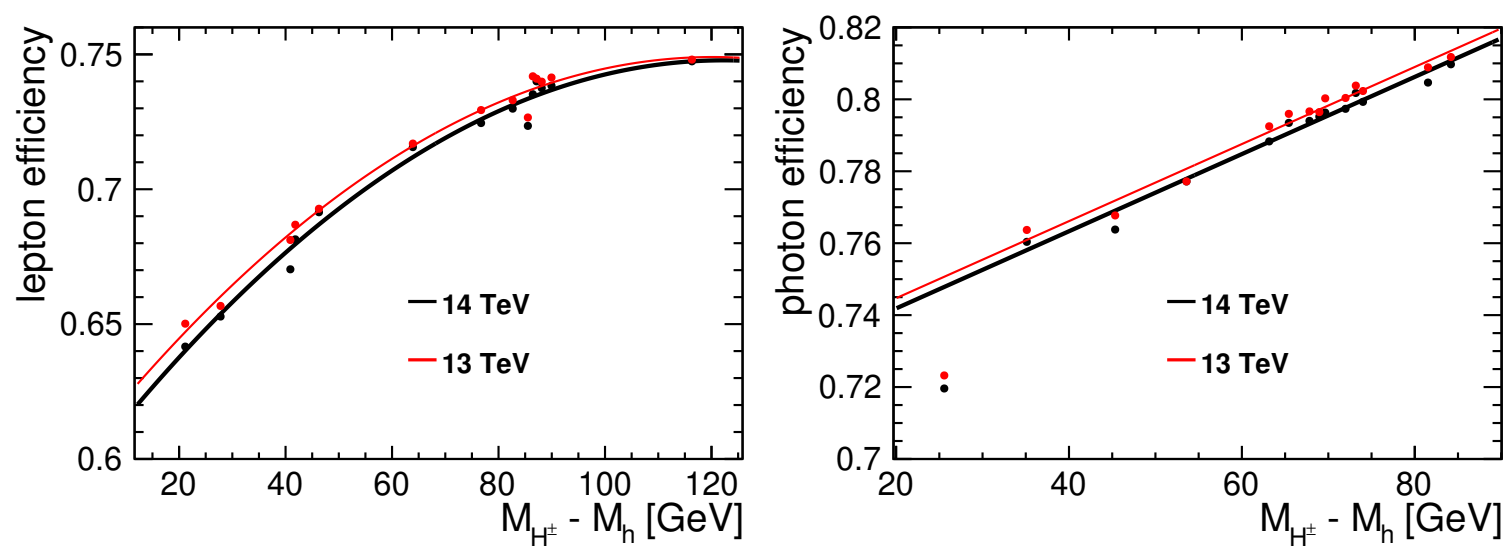

Figure 4. The lepton tagging efficiency (left) and the photon tagging efficiency (right) are shown as a function of the mass difference between the $H^{ \pm}$and $h$ states.

a lepton in the final state can be successfully reconstructed and recognised, while for the photon case we count four photons total efficiencies, then convert to single photon efficiency. The efficiencies for our BPs are shown in figure 4. By using some simple functions to fit the curves in figure 4, we find that the efficiencies for leptons and photons can be expressed by the following two relations, respectively: ${ }^{2}$

$$
\begin{aligned}
& \epsilon_{\ell}=\left\{\begin{array}{l}
0.59035+0.002574 x-1.0523 \times 10^{-5} x^{2}, \text { where } x=\left(M_{H^{ \pm}}-M_{h}\right)(14 \mathrm{TeV}), \\
0.59892+0.002500 x-1.0373 \times 10^{-5} x^{2}, \text { where } x=\left(M_{H^{ \pm}}-M_{h}\right)(13 \mathrm{TeV}),
\end{array}\right. \\
& \epsilon_{\gamma}=\left\{\begin{array}{l}
0.001073 \times M_{h}+0.72040,(14 \mathrm{TeV}), \\
0.001072 \times M_{h}+0.72327,(13 \mathrm{TeV}) .
\end{array}\right.
\end{aligned}
$$

Their shapes are captured in figure 4 .

\footnotetext{
${ }^{2}$ We should mention here that, to parameterise the typical photon efficiency, we used all points except BP1, which has evidently a smaller efficiency that the other BPs, because $m_{h}$ is about $25 \mathrm{GeV}$, which in turn means that the rate for BP1 will be somewhat overestimated.
} 
$13 \mathrm{TeV}$
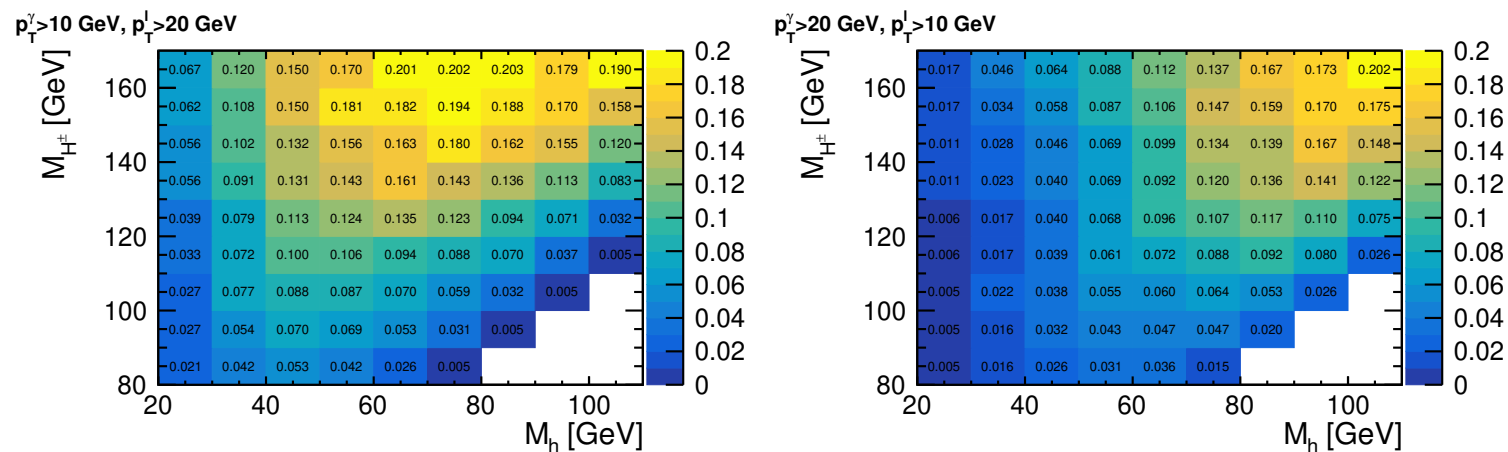

$14 \mathrm{TeV}$
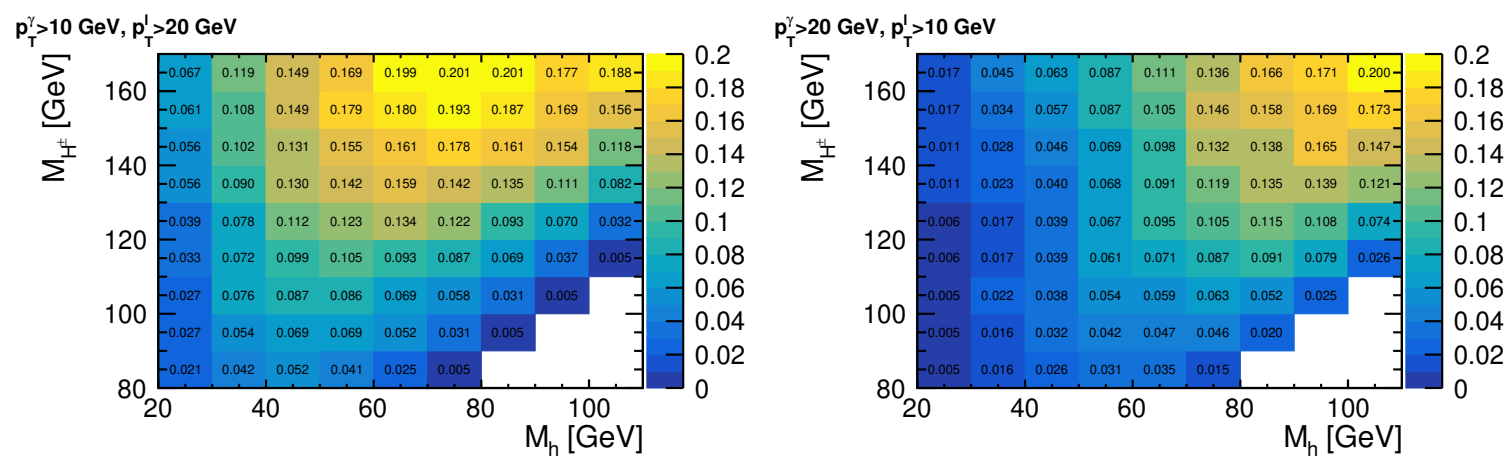

Figure 5. Fiducial efficiency $\epsilon$ for detecting the signal via the $\ell \nu_{\ell}+4 \gamma$ signature at detector level for the two sets of cuts given in eqs. (3.4) (left) and (3.5) (right).

Thus, we can derive the acceptance efficiency at detector level, $\epsilon_{\text {det }}$, which can be expressed as a function of the mistagging rate of jets $\left(10^{-3}\right)$, lepton reconstruction $\epsilon_{\ell}$ and photon detection efficiencies $\epsilon_{\gamma}$ as

$$
\epsilon_{\mathrm{det}}=10^{-3 n_{j}} \times \epsilon_{\ell} \times \epsilon_{\gamma}^{4-n_{j}},
$$

where $n_{j}$ denotes the jet number and $4-n_{j}$ denotes the photon number. By using this acceptance efficiency at detector level, we can estimate the detection efficiency for the whole parameter space of our 2HDM Type-I scenario.

Since the $W^{ \pm}$bosons in the final state could be either on-shell or off-shell, depending on the BP, in this work, we adopt two sets of cuts (see ref. [1]) to examine how the efficiencies can change. The first set of cuts is

$$
p_{T}^{\gamma}>10 \mathrm{GeV}, \quad p_{T}^{\ell}>20 \mathrm{GeV},
$$

and the second set of cuts is

$$
p_{T}^{\gamma}>20 \mathrm{GeV}, \quad p_{T}^{\ell}>10 \mathrm{GeV} .
$$




\begin{tabular}{|c|c|c|c|}
\hline Process & Cross section $(\mathrm{fb})$ & After selection & Generated event number \\
\hline$W^{ \pm}+4 j 0 \gamma$ & 145890 & 0 & $1 \times 10^{6}$ \\
\hline$W^{ \pm}+3 j 1 \gamma$ & 1730 & 0 & $1 \times 10^{6}$ \\
\hline$W^{ \pm}+2 j 2 \gamma$ & 10.2 & $2.55 \times 10^{-4}$ & $2 \times 10^{6}$ \\
\hline$W^{ \pm}+1 j 3 \gamma$ & 0.0282 & $1.52 \times 10^{-4}$ & $1 \times 10^{6}$ \\
\hline$W^{ \pm}+0 j 4 \gamma$ & $1.69 \times 10^{-5}$ & $5.71 \times 10^{-6}$ & $1 \times 10^{6}$ \\
\hline
\end{tabular}

Table 4. The cross sections of background processes with $\sqrt{s}=13 \mathrm{TeV}$ are given, after taking into account cuts and detector effects.

To determine the fiducial efficiencies of each point in the parameter space, we use the relation

$$
\epsilon=\sigma(\text { cuts }) \times \epsilon_{\mathrm{det}} / \sigma(\text { no cuts }) .
$$

The results for the fiducial efficiencies are shown in figure 5 for the two sets of cuts, which show a strong dependence on the masses of both charged and neutral Higgs boson. Obviously, in the parameter region where the $W^{ \pm}$is on-shell and the $h$ not too light, a larger efficiency can be obtained. In contrast, in the parameter space region with a very light $h$ (say $M_{h}$ around $25 \mathrm{GeV}$ ), the signal loss is caused by soft photons while, in the parameter space region with $M_{H^{ \pm}}-M_{h}$ small, the signal loss is caused by soft leptons. When comparing our two sets of cuts, we can see that the first one has a better acceptance efficiency than the second one in covering a wider region of parameter space.

Before moving on to compute the significances of our signal for the BPs introduced, we present table 4 for the purpose of confirming the statement made in ref. [1], that none of the backgrounds is really observable for any realistic LHC and HL-LHC luminosity. (Results are shown here for $13 \mathrm{TeV}$, but the conclusion is the same for $14 \mathrm{TeV}$.) We also present the predicted cross sections for the signals emerging from the BPs after taking into account the cuts and the detector acceptance efficiency in tables 5 and 6 , where we have considered $\sqrt{s}=13$ and $14 \mathrm{TeV}$, respectively. Due to the fact that the quark fluxes cannot be greatly enhanced when the collision energy increases from 13 to $14 \mathrm{TeV}$, we notice that the cross sections of the signal processes can only increase by $5 \%$ to $10 \%$ between the lower and higher center-of-mass energies.

There is one remark that ought to be made at this point, on the number of events used in our MC analysis for the signal and background processes. Considering that the cross section of the signal process and those of the dominant backgrounds $W+2 j 2 \gamma, W+1 j 3 \gamma$ and $W+4 \gamma$ are $1 \sim 10 \mathrm{fb}, 10.2 \mathrm{fb}, 0.028 \mathrm{fb}$ and $1.7 \times 10^{-5} \mathrm{fb}$, respectively, we believe that the number of $\mathrm{MC}$ points generated, $10^{6}$ or higher, is good enough to avoid large fluctuations in statistics. Further, while it is clear that the cross sections of the background processes $W+4 j$ and $W+3 j \gamma$ are extremely large, after taking into account the fake photon rate for a jet $\left(10^{-3}\right)$ (and using $W+2 j 2 \gamma$ as a reference process), it is reasonable to estimate that these two processes would contribute $10^{-5}-10^{-6} \mathrm{fb}$ at most, which are thus presented as zeroes in table IV. (Indeed, these results are in line with what was estimated in [1].) 


\begin{tabular}{|c|c|c|c|}
\hline Cross section $(\mathrm{fb})$ & MG & After selection & Estimate \\
\hline BP1 & 2.09 & 0.42 & 0.49 \\
\hline BP2 & 7.43 & 1.89 & 1.87 \\
\hline BP3 & 0.57 & 0.15 & 0.15 \\
\hline BP4 & 1.17 & 0.29 & 0.30 \\
\hline BP5 & 9.49 & 2.57 & 2.55 \\
\hline BP6 & 12.48 & 3.65 & 3.60 \\
\hline BP7 & 10.42 & 2.90 & 2.90 \\
\hline BP8 & 8.09 & 2.18 & 2.16 \\
\hline BP9 & 7.31 & 1.98 & 1.95 \\
\hline BP10 & 4.72 & 1.47 & 1.47 \\
\hline BP11 & 4.65 & 1.39 & 1.38 \\
\hline BP12 & 4.59 & 1.39 & 1.39 \\
\hline BP13 & 4.36 & 1.34 & 1.33 \\
\hline BP14 & 4.23 & 1.34 & 1.35 \\
\hline
\end{tabular}

Table 5. The cross sections of our signal processes with $\sqrt{s}=13 \mathrm{TeV}$ are given, after taking into account cuts and detector effects (we also show the results from our analytical estimate).

\begin{tabular}{|c|c|c|c|}
\hline Cross section $(\mathrm{fb})$ & MG & After selection & Estimate \\
\hline BP1 & 2.27 & 0.44 & 0.52 \\
\hline BP2 & 8.02 & 2.00 & 1.99 \\
\hline BP3 & 0.62 & 0.15 & 0.16 \\
\hline BP4 & 1.27 & 0.31 & 0.32 \\
\hline BP5 & 10.19 & 2.68 & 2.68 \\
\hline BP6 & 13.46 & 3.86 & 3.81 \\
\hline BP7 & 11.26 & 3.10 & 3.07 \\
\hline BP8 & 8.76 & 2.30 & 2.29 \\
\hline BP9 & 7.87 & 2.09 & 2.05 \\
\hline BP10 & 5.16 & 1.59 & 1.58 \\
\hline BP11 & 5.03 & 1.48 & 1.47 \\
\hline BP12 & 4.93 & 1.47 & 1.46 \\
\hline BP13 & 4.76 & 1.44 & 1.43 \\
\hline BP14 & 4.61 & 1.43 & 1.44 \\
\hline
\end{tabular}

Table 6. The cross sections of our signal processes with $\sqrt{s}=14 \mathrm{TeV}$ are given, after taking into account cuts and detector effects (we also show the results from our analytical estimate). 


\begin{tabular}{|c|c|c|c|c|c|c|c|c|c|c|c|c|c|c|}
\hline BPs & 1 & 2 & 3 & 4 & 5 & 6 & 7 & 8 & 9 & 10 & 11 & 12 & 13 & 14 \\
\hline$\sigma_{13 \mathrm{TeV}}$ & 12.1 & 23.7 & 6.7 & 9.4 & 27.4 & 32.6 & 29.2 & 25.2 & 23.9 & 20.8 & 20.2 & 20.3 & 19.9 & 19.9 \\
\hline$\sigma_{14 \mathrm{TeV}}$ & 12.5 & 24.4 & 7.0 & 9.8 & 28.4 & 33.9 & 30.3 & 26.2 & 24.8 & 21.8 & 21.1 & 21.0 & 20.8 & 20.8 \\
\hline
\end{tabular}

Table 7. The significances for all $14 \mathrm{BPs}$ at the LHC are tabulated, where the luminosity is assumed to be $300 \mathrm{fb}^{-1}$ at both $\sqrt{s}=13$ and $14 \mathrm{TeV}$.

To compute the significances, due to the tiny number of background events, we can neglect the latter safely. Therefore, the predicted significances can be computed by using the relation $\frac{N_{S}}{\sqrt{N_{S}+N_{B}}} \sim \sqrt{N_{S}}$. The corresponding results are shown in table 7 . We find that the predicted significances are larger than 5 for all 14 BPs in our 2HDM Type-I scenario when the luminosity is assumed to be $300 \mathrm{fb}^{-1}$, both at $\sqrt{s}=13$ and $14 \mathrm{TeV}$. The predicted significances for both energies and the given luminosity over the $\left(M_{h}, M_{H^{ \pm}}\right)$plane are shown in figure 6 , which are obtained from the described convolution of production cross sections with cut and acceptance efficiencies at detector level. To obtain the results given here, for each point on the $\left(M_{h}, M_{H^{ \pm}}\right)$plane, we allow $\tan \beta$ and $\sin (\beta-\alpha)$ to vary and take the maximal significance. In such a figure, it should be pointed out that, when $m_{h}<62.5 \mathrm{GeV}$, the decay mode $H \rightarrow h h$ of the SM-like Higgs boson $H$ is open. Therefore, fewer points in the parameter space are allowed by the Higgs boson data collected at the LHC, so that the predicted significances drop when $M_{h}$ reaches the value of $62.5 \mathrm{GeV}$. Another remarkable feature is that the predicted significances are larger than 5 for nearly all points on the $\left(M_{h}, M_{H^{ \pm}}\right)$plane. In fact, only very few points on the grid have a significance below 2 . Hence, in short, the whole interesting parameter space of our 2HDM Type-I can be either ruled out or discovered by the accumulated datasets of the entire Run 3 and/or only 1/10 of the HL-LHC one.

The predicted significances in term of the 2HDM Type-I parameter space mapped over the $(\sin (\beta-\alpha), \tan \beta)$ plane are shown in figure 7 for both $\sqrt{s}=13$ and $14 \mathrm{TeV}$, respectively. To obtain the results given here, for each point on the $(\sin (\beta-\alpha), \tan \beta)$ plane, we allow $M_{H^{ \pm}}$and $M_{h}$ to vary and take again the maximal significance. This mapping makes it clear that some amount of fine-tuning in $\sin (\beta-\alpha)$ and/or $\tan \beta$ is necessary to obtain large significances. However, for any $\tan \beta>5$, there is always a choice of $\sin (\beta-\alpha)$ that allows one to make a definite statement at both the LHC stages considered on the portion of parameter space of the 2HDM Type-I that we have sampled.

We conclude our numerical analysis by noting that, altogether, as we can observe from figures $6-7$, it is the first set of cuts given in eq. (3.4) that yields better significances than the one in eq. (3.5).

\section{Conclusions}

In this paper, we have examined the feasibility of the signature $W^{ \pm}+4 \gamma$, where the $W^{ \pm}$ decays leptonically in electrons and muons, from the associated production of the charged Higgs boson and lightest neutral Higgs state of the 2HDM Type-I (i.e., via $p p \rightarrow H^{ \pm} h \rightarrow$ $\left.W^{ \pm(*)} h h \rightarrow \ell \nu_{\ell}+4 \gamma\right)$ at the LHC with a collision energy of $\sqrt{s}=13$ and $14 \mathrm{TeV}$ and 
$13 \mathrm{TeV}$
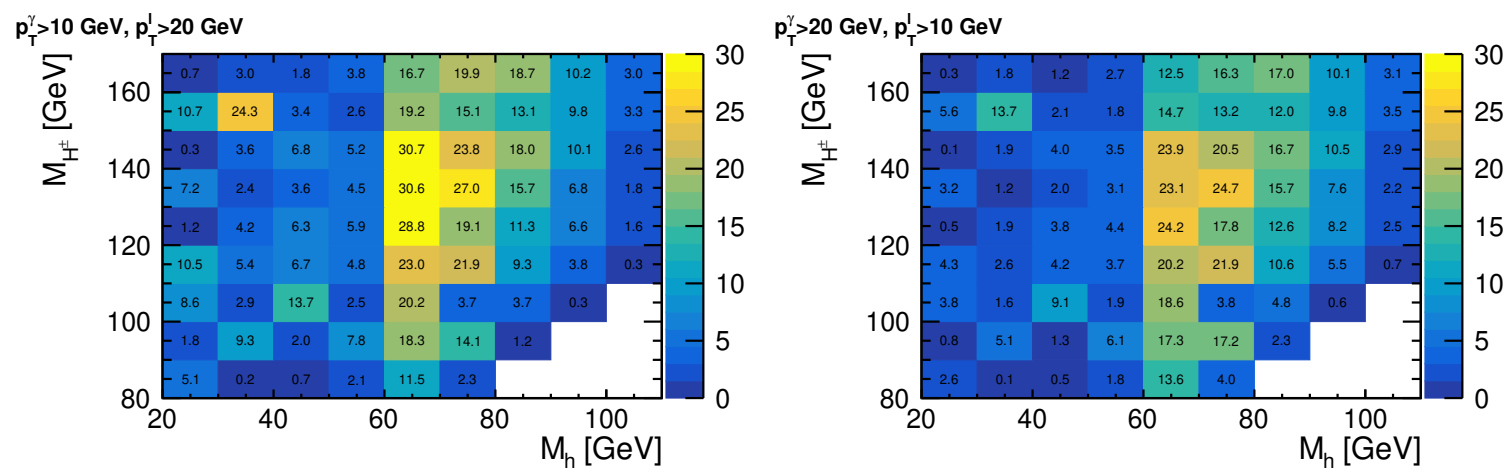

$14 \mathrm{TeV}$
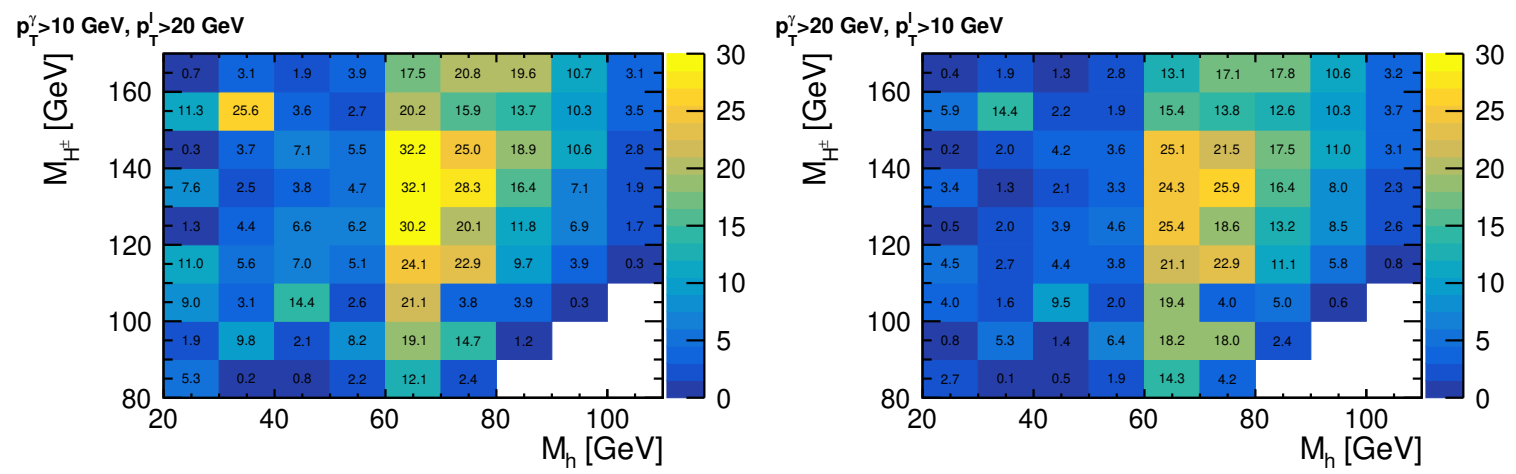

Figure 6. The predicted significances over the $\left(M_{h}, M_{H^{ \pm}}\right)$plane for the two sets of cuts given in eqs. (3.4) (left) and (3.5) (right), when $\sqrt{s}=13 \mathrm{TeV}$ (top) as well as $\sqrt{s}=14 \mathrm{TeV}$ (bottom) and $L=300 \mathrm{fb}^{-1}$.

an integrated luminosity of $L=300 \mathrm{fb}^{-1}$. Our analysis has been a detector level study exploiting full MC event generation including parton shower, hadronisation and heavy flavour decays. By doing so, we have confirmed a previous study done solely at the parton level, as we have proven that, even in presence of background generated by both real and fake photons (from jets), the signal is essentially background free, so that significances only depend upon the signal cross sections and the collider integrated luminosities. We have also provided some reliable estimates for the detector efficiency and associated heat maps which can expedite an estimate of the signal significance over the relevant 2HDM Type-I parameter space, which we deem useful for current LHC working groups. Finally, for more thorough experimental analyses, we have also published $14 \mathrm{BPs}$, where the $W^{ \pm}$boson can be either on-shell or off-shell, depending on the mass difference $M_{H^{ \pm}}-M_{h}$.

\section{Acknowledgments}

The work of AA, RB, MK and BM is supported by the Moroccan Ministry of Higher Education and Scientific Research MESRSFC and CNRST Project PPR/2015/6. The work of SM is supported in part through the NExT Institute and the STFC Consolidated Grant No. ST/L000296/1. Y. W. is supported by the 'Scientific Research Funding Project 
$13 \mathrm{TeV}$
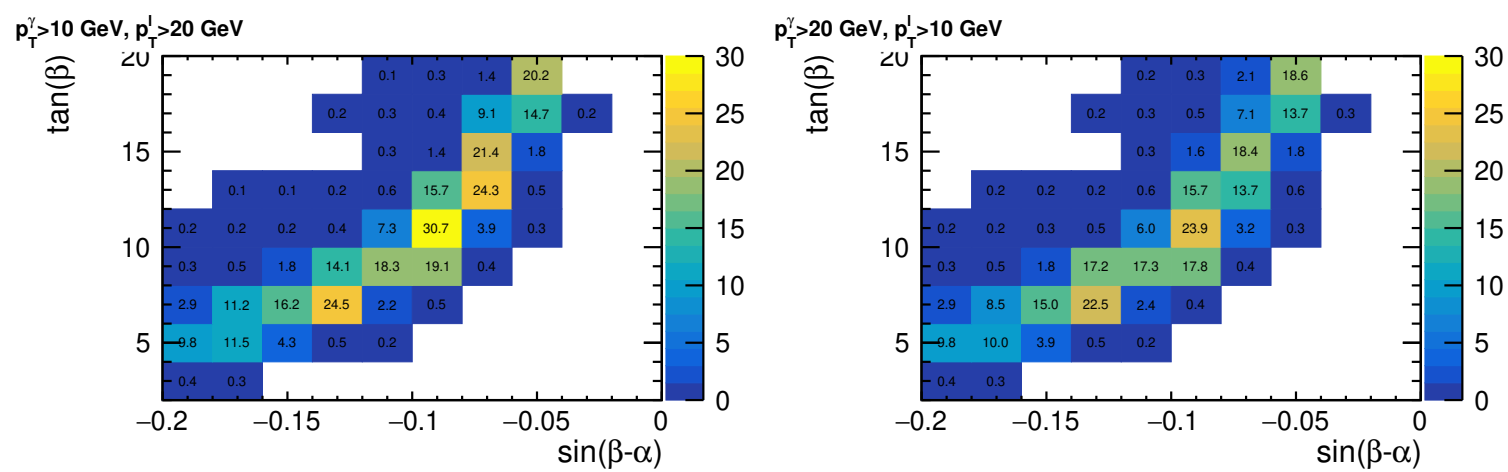

$14 \mathrm{TeV}$
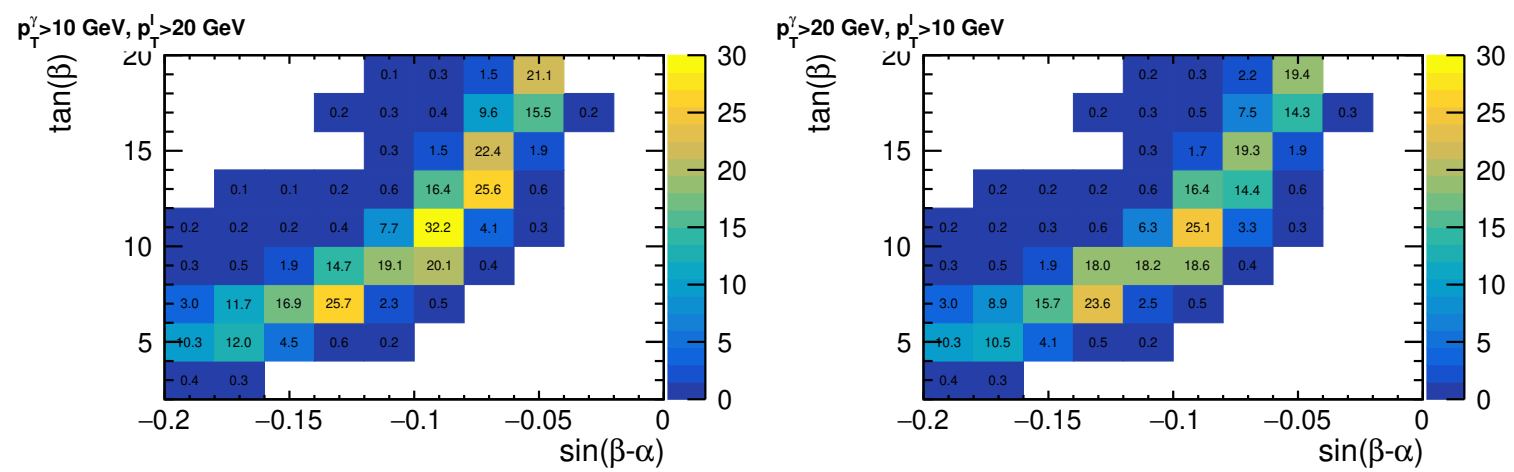

Figure 7. The predicted significances over the $(\sin (\beta-\alpha), \tan \beta)$ plane for the two sets of cuts given in eqs. (3.4) (left) and (3.5) (right), when $\sqrt{s}=13 \mathrm{TeV}$ (top) as well as $\sqrt{s}=14 \mathrm{TeV}$ (bottom) and $L=300 \mathrm{fb}^{-1}$.

for Introduced High-level Talents' of the Inner Mongolia Normal University Grant No. 2019YJRC001, and the scientific research funding for introduced high-level talents of Inner Mongolia of China. Q.-S. Yan's work is supported by the Natural Science Foundation of China Grant No. 11875260.

Open Access. This article is distributed under the terms of the Creative Commons Attribution License (CC-BY 4.0), which permits any use, distribution and reproduction in any medium, provided the original author(s) and source are credited.

\section{References}

[1] A. Arhrib, R. Benbrik, R. Enberg, W. Klemm, S. Moretti and S. Munir, Identifying a light charged Higgs boson at the LHC run II, Phys. Lett. B 774 (2017) 591 [arXiv:1706. 01964] [INSPIRE].

[2] F. Gianotti et al., Physics potential and experimental challenges of the LHC luminosity upgrade, Eur. Phys. J. C 39 (2005) 293 [hep-ph/0204087] [INSPIRE].

[3] X. Cid Vidal et al., Report from working group 3: beyond the Standard Model physics at the HL-LHC and HE-LHC, CERN Yellow Rep. Monogr. 7 (2019) 585 [arXiv:1812.07831] [INSPIRE]. 
[4] G.C. Branco, P.M. Ferreira, L. Lavoura, M.N. Rebelo, M. Sher and J.P. Silva, Theory and phenomenology of two-Higgs-doublet models, Phys. Rept. 516 (2012) 1 [arXiv:1106.0034] [INSPIRE].

[5] A.G. Akeroyd, A. Arhrib and E.-M. Naimi, Note on tree level unitarity in the general two Higgs doublet model, Phys. Lett. B 490 (2000) 119 [hep-ph/0006035] [INSPIRE].

[6] J.F. Gunion and H.E. Haber, The CP conserving two Higgs doublet model: the approach to the decoupling limit, Phys. Rev. D 67 (2003) 075019 [hep-ph/0207010] [INSPIRE].

[7] M.E. Peskin and T. Takeuchi, Estimation of oblique electroweak corrections, Phys. Rev. D 46 (1992) 381 [INSPIRE].

[8] Particle Data Group collaboration, Review of particle physics, Chin. Phys. C 38 (2014) 090001 [INSPIRE].

[9] P. Bechtle et al., HiggsBounds-5: testing Higgs sectors in the LHC 13 TeV era, Eur. Phys. J. C 80 (2020) 1211 [arXiv: 2006.06007] [INSPIRE].

[10] P. Bechtle, S. Heinemeyer, T. Klingl, T. Stefaniak, G. Weiglein and J. Wittbrodt, HiggsSignals-2: probing new physics with precision Higgs measurements in the LHC $13 \mathrm{TeV}$ era, Eur. Phys. J. C 81 (2021) 145 [arXiv: 2012.09197] [InSPIRE].

[11] HFLAV collaboration, Averages of b-hadron, c-hadron, and $\tau$-lepton properties as of summer 2016, Eur. Phys. J. C 77 (2017) 895 [arXiv: 1612.07233] [INSPIRE].

[12] LHCb collaboration, Measurement of the $B_{s}^{0} \rightarrow \mu^{+} \mu^{-}$branching fraction and effective lifetime and search for $B^{0} \rightarrow \mu^{+} \mu^{-}$decays, Phys. Rev. Lett. 118 (2017) 191801 [arXiv: 1703.05747] [INSPIRE].

[13] F. Mahmoudi, SuperIso v2.3: a program for calculating flavor physics observables in supersymmetry, Comput. Phys. Commun. 180 (2009) 1579 [arXiv:0808.3144] [INSPIRE].

[14] D. Eriksson, J. Rathsman and O. Stal, 2HDMC: two-Higgs-doublet model calculator physics and manual, Comput. Phys. Commun. 181 (2010) 189 [arXiv:0902.0851] [INSPIRE].

[15] DELPHI collaboration, Search for fermiophobic Higgs bosons in final states with photons at LEP 2, Eur. Phys. J. C 35 (2004) 313 [hep-ex/0406012] [InSPIRE].

[16] LEP collaboration, Fermiophobic Higgs bosons at LEP, Nucl. Phys. B Proc. Suppl. 117 (2003) 743 [hep-ex/0212038] [INSPIRE].

[17] D0 collaboration, Search for decay of a fermiophobic Higgs boson $h(f) \rightarrow \gamma \gamma$ with the D0 detector at $\sqrt{s}=1.96 \mathrm{TeV}$, Phys. Rev. Lett. 101 (2008) 051801 [arXiv:0803.1514] [INSPIRE].

[18] P. Bechtle et al., HiggsBounds-4: improved tests of extended Higgs sectors against exclusion bounds from LEP, the Tevatron and the LHC, Eur. Phys. J. C 74 (2014) 2693 [arXiv: 1311.0055] [INSPIRE].

[19] OPAL collaboration, Search for associated production of massive states decaying into two photons in $e^{+} e^{-}$annihilations at $\sqrt{s}=88 \mathrm{GeV}$ to $209 \mathrm{GeV}$, Phys. Lett. B 544 (2002) 44 [hep-ex/0207027] [INSPIRE].

[20] A.G. Akeroyd and M.A. Diaz, Searching for a light fermiophobic Higgs boson at the Tevatron, Phys. Rev. D 67 (2003) 095007 [hep-ph/0301203] [INSPIRE].

[21] A.G. Akeroyd, A. Alves, M.A. Diaz and O.J.P. Eboli, Multi-photon signatures at the Fermilab Tevatron, Eur. Phys. J. C 48 (2006) 147 [hep-ph/0512077] [InSPIRE]. 
[22] CDF collaboration, Search for a low-mass neutral Higgs boson with suppressed couplings to fermions using events with multiphoton final states, Phys. Rev. D 93 (2016) 112010 [arXiv: 1601.00401] [INSPIRE].

[23] A.G. Akeroyd, M.A. Diaz and F.J. Pacheco, Double fermiophobic Higgs boson production at the CERN LHC and LC, Phys. Rev. D 70 (2004) 075002 [hep-ph/0312231] [INSPIRE].

[24] A. Delgado, M. Garcia-Pepin, M. Quirós, J. Santiago and R. Vega-Morales, Diphoton and diboson probes of fermiophobic Higgs bosons at the LHC, JHEP 06 (2016) 042 [arXiv: 1603. 00962] [INSPIRE].

[25] A. Arhrib et al., New discovery modes for a light charged Higgs boson at the LHC, JHEP 10 (2021) 073 [arXiv: 2106.13656] [INSPIRE].

[26] A. Arhrib, R. Benbrik and S. Moretti, Bosonic decays of charged Higgs bosons in a 2HDM type-I, Eur. Phys. J. C 77 (2017) 621 [arXiv:1607.02402] [inSPIRE].

[27] H. Bahl, T. Stefaniak and J. Wittbrodt, The forgotten channels: charged Higgs boson decays to a $W^{ \pm}$and a non-SM-like Higgs boson, JHEP 06 (2021) 183 [arXiv:2103.07484] [INSPIRE].

[28] J. Alwall et al., The automated computation of tree-level and next-to-leading order differential cross sections, and their matching to parton shower simulations, JHEP 07 (2014) 079 [arXiv: 1405.0301] [INSPIRE].

[29] T. Sjöstrand, S. Mrenna and P.Z. Skands, PYTHIA 6.4 physics and manual, JHEP 05 (2006) 026 [hep-ph/0603175] [inSPIRE].

[30] DELPHES 3 collaboration, DELPHES 3, a modular framework for fast simulation of a generic collider experiment, JHEP 02 (2014) 057 [arXiv:1307.6346] [INSPIRE].

[31] M. Cacciari, G.P. Salam and G. Soyez, The anti- $k_{t}$ jet clustering algorithm, JHEP 04 (2008) 063 [arXiv:0802.1189] [INSPIRE].

[32] M. Cacciari, G.P. Salam and G. Soyez, FastJet user manual, Eur. Phys. J. C 72 (2012) 1896 [arXiv:1111.6097] [INSPIRE].

[33] S. Catani, Y.L. Dokshitzer, M.H. Seymour and B.R. Webber, Longitudinally invariant $K_{t}$ clustering algorithms for hadron hadron collisions, Nucl. Phys. B 406 (1993) 187 [InSPIRE].

[34] Y.L. Dokshitzer, G.D. Leder, S. Moretti and B.R. Webber, Better jet clustering algorithms, JHEP 08 (1997) 001 [hep-ph/9707323] [INSPIRE].

[35] M. Wobisch and T. Wengler, Hadronization corrections to jet cross-sections in deep inelastic scattering, in Workshop on Monte Carlo generators for HERA physics (plenary starting meeting), (1998), pg. 270 [hep-ph/9907280] [INSPIRE].

[36] ATLAS collaboration, Reconstruction, energy calibration, and identification of hadronically decaying tau leptons in the ATLAS experiment for run-2 of the LHC, Tech. Rep.

ATL-PHYS-PUB-2015-045, CERN, Geneva, Switzerland (2015). 